\title{
Chapter 15 \\ On the Coalgebraic Theory of Kleene Algebra with Tests
}

\author{
Dexter Kozen
}

In honor of Rohit Parikh

\begin{abstract}
We develop a coalgebraic theory of Kleene algebra with tests (KAT) along the lines of Rutten (1998) for Kleene algebra (KA) and Chen and Pucella (Electron Notes Theor Comput Sci 82(1), 2003) for a limited version of KAT, resolving some technical issues raised by Chen and Pucella. Our treatment includes a simple definition of the Brzozowski derivative for KAT expressions and an automata-theoretic interpretation involving automata on guarded strings. We also give a complexity analysis, showing that an efficient implementation of coinductive equivalence proofs in this setting is tantamount to a standard automata-theoretic construction. It follows that coinductive equivalence proofs can be generated automatically in PSPACE. This matches the bound of Worthington (2008) for the automatic generation of equational proofs in KAT.
\end{abstract}

Keywords Kleene algebra $\cdot$ Kleene algebra with tests $\cdot$ Coalgebra $\cdot$ Verification

\subsection{Introduction}

Kleene algebra (KA) is the algebra of regular expressions. The operations,$+ \cdot$, and ${ }^{*}$ of Kleene algebra can be used to model nondeterministic choice, sequential composition, and iteration, respectively, on a set of actions.

Kleene algebra with tests (KAT) is an extension of KA obtained by identifying a subset of tests, which must satisfy the axioms of Boolean algebra as well. The

D. Kozen $(\varangle)$

Computer Science Department, Cornell University, Ithaca, NY 14853-7501, USA

e-mail: kozen@cs.cornell.edu

(C) Springer International Publishing AG 2017

C. Başkent et al. (eds.), Rohit Parikh on Logic, Language and Society,

Outstanding Contributions to Logic 11, DOI 10.1007/978-3-319-47843-2_15 
two sorts, actions and tests, interact seamlessly: on tests, nondeterministic choice becomes join and sequential composition becomes meet.

The presence of tests allows KAT to model basic programming and verification constructs such as conditional tests, while loops, and Hoare triples. Thus KAT gives a simple equational approach to partial correctness. By now KAT has a well-developed theory, including an extensive model theory and results on expressiveness, deductive completeness, and complexity. It has been applied successfully in a number of areas, including the verification of compiler optimizations and communication protocols and various other program analysis tasks.

Traditionally, KAT is axiomatized equationally (Kozen 1997). In Chen and Pucella (2003), Chen and Pucella develop a coalgebraic theory of KAT inspired by Rutten's coalgebraic theory of KA based on deterministic automata (Rutten 1998). Remarking that "the known automata-theoretic presentation of KAT (Kozen 2003) does not lend itself to a coalgebraic theory," and that "the notion of derivative, essential to the coinduction proof principle in this context, is not readily definable for KAT expressions as defined in Kozen (1997)," Chen and Pucella develop a new interpretation of KAT expressions and a corresponding automata theory differing from Kozen (2003) in several respects. They give a coinductive proof principle and show how it can be used to establish equivalence of expressions. This gives an alternative to equational proofs using the standard axiomatization (Kozen 1997) or by minimization of deterministic automata (Kozen 2003).

The ability to generate equivalence proofs automatically has important implications for proof-carrying code. Chen and Pucella argue that the coalgebraic approach makes this possible, since proofs can be generated purely mechanically via repeated application of the Brzozowski derivative, whereas classical equational logic "requires creativity" (Chen and Pucella 2003). This is not strictly true, as Worthington (Worthington 2008) has shown that equational proofs can also be generated automatically. However, it is fair to say that the coinductive approach does provide a more natural mechanism.

Still unresolved is the issue of proof complexity in the coinductive setting. Chen and Pucella claim that coinduction can give shorter proofs, but they give no supporting evidence. Worthington's technique is known to require PSPACE and to produce exponential-size proofs in the worst case. This worst-case bound is unlikely to be significantly improved, as the equational theory of KAT is PSPACE-complete (Cohen et al. 1996).

Chen and Pucella's treatment has a few technical shortcomings, as they themselves point out. In their words:

The "path independence" of a mixed automaton gives any mixed automaton a certain form of redundancy. This redundancy persists in the definition of bisimulation...An open question is to cleanly eliminate this redundancy; a particular motivation for doing this would be to make proofs of expression equivalence as simple as possible. Along these lines, it would be of interest to develop other weaker notions of bisimulation that give rise to bisimulations; pseudo-bisimulations require a sort of "fixed variable ordering" that does not seem absolutely necessary... 
Another issue for future work would be to give a class of expressions wider than our mixed expressions for which there are readily understandable and applicable rules for computing derivatives. In particular, a methodology for computing derivatives of the KAT expressions defined by Kozen (1997) would be nice to see. Intuitively, there seems to be a tradeoff between the expressiveness of the regular expression language and the simplicity of computing derivatives (in the context of KAT). Formal tools for understanding this tradeoff could potentially be quite useful (Chen and Pucella 2003).

This paper addresses these issues. We develop a coalgebraic theory of KAT, which we call KCT, along the lines of Chen and Pucella (2003); Rutten (1998). Our treatment includes a new definition of the Brzozowski derivative, but in the context of the original automata-theoretic formulation of KAT involving automata on guarded strings (Kozen 2003). The syntactic form of the Brzozowski derivative applies to all KAT expressions as defined in Kozen (1997). The somewhat artificial concepts of path independence, fixed variable ordering, and pseudo-bisimulation do not arise in this setting. This treatment places KCT within the general coalgebraic framework described by Bonsangue et al. (2007, 2009).

We also give a complexity analysis of the coinductive proof principle. We show that an efficient implementation is tantamount to the construction of nondeterministic automata from the given expressions by a Kleene construction, determinizing the two automata by a standard subset construction, and constructing a bisimulation on states of the resulting deterministic automata. It follows that coinductive equivalence proofs can be generated automatically in PSPACE. This matches Worthington's bound (Worthington 2008) for equational proofs.

\subsection{KA and KAT}

\subsubsection{Kleene Algebra}

Kleene algebra (KA) is the algebra of regular expressions (Conway 1971; Kleene 1956). The axiomatization used here is from Kozen (1994). A Kleene algebra is a structure $\left(K,+, \cdot{ }^{*}, 0,1\right)$ such that $K$ is an idempotent semiring under $+, \cdot, 0$, and 1 and satisfies the axioms

$$
\begin{array}{rlrl}
1+p p^{*} & \leq p^{*} & q+p r & \leq r \Rightarrow p^{*} q \leq r \\
1+p^{*} p \leq p^{*} & q+r p \leq r \Rightarrow q p^{*} \leq r
\end{array}
$$

for ${ }^{*}$. There is a natural partial order $p \leq q \stackrel{\text { def }}{\Longleftrightarrow} p+q=q$.

Standard models include the family of regular sets over a finite alphabet, the family of binary relations on a set, and the family of $n \times n$ matrices over another Kleene algebra. Other more unusual interpretations include the min,+ algebra, also known as the tropical semiring, used in shortest path algorithms, and models consisting of convex polyhedra used in computational geometry. 
The completeness result of Kozen (1994) says that the algebra of regular sets of strings over a finite alphabet $\Sigma$ is the free Kleene algebra on generators $\Sigma$. The axioms are also complete for the equational theory of relational models.

\subsubsection{Kleene Algebra with Tests}

A Kleene algebra with tests (KAT) (Kozen 1997) consists of a Kleene algebra $K$ with an embedded Boolean algebra $B$ such that the semiring structure on $B$ is a subalgebra of the semiring structure on $K$. Elements of $B$ are called tests. The Boolean negation operator is defined only on tests.

Like KA, KAT has language and relational models and is deductively complete over these interpretations (Kozen and Smith 1996). The chief language-theoretic models are the regular sets of guarded strings over alphabets $\Sigma$ and $T$ of primitive action and test symbols, respectively (see Sect. 15.3.1). This is the free KAT on generators $\Sigma, T$. The set of guarded strings represented by a KAT expression $e$ is denoted $\mathrm{GS}(e)$.

KAT can code elementary programming constructs and Hoare partial correctness assertions and subsumes propositional Hoare logic (PHL). It is deductively complete over relational models, whereas $\mathrm{PHL}$ is not. Moreover, KAT is no more difficult to decide, as PHL, KA, and KAT are all PSPACE-complete.

For KAT expressions $e_{1}, e_{2}$, we write $e_{1} \leq e_{2}$ if this inequality holds in the free KAT on generators $\Sigma, T$; that is, if it is a consequence of the axioms of KAT.

See Kozen $(1994,1997,2000)$ for a more detailed introduction.

\subsection{Automata on Guarded Strings}

Automata on guarded strings (AGS), also known as automata with tests, were introduced in Kozen (2003). They are a generalization of ordinary finite-state automata to include tests. An ordinary automaton with null transitions is an AGS over the two-element Boolean algebra.

\subsubsection{Guarded Strings}

Guarded strings were first introduced in Kaplan (1969). A guarded string over $\Sigma, T$ is an alternating sequence $\alpha_{0} p_{1} \alpha_{1} p_{2} \alpha_{2} \cdots p_{n} \alpha_{n}, n \geq 0$, where $p_{i} \in \Sigma$ and the $\alpha_{i}$ are atoms (minimal nonzero elements) of the free Boolean algebra $B$ generated by $T$. The set of atoms is denoted At. The elements of At can be regarded either as conjunctions of literals of $T$ (elements of $T$ or their negations) or as truth assignments to $T$. A guarded string is thus an element of At $\cdot(\Sigma \cdot \mathrm{At})^{*}$. The set of all guarded strings is 
denoted GS. Guarded strings represent the join-irreducible elements of the free KAT on generators $\Sigma, T$.

\subsubsection{Nondeterministic Automata}

A nondeterministic AGS consists of a labeled directed graph with two types of transitions, action transitions labeled with elements of $\Sigma$ and test transitions labeled with elements of $B$. There is a set start of start states and a set accept of accept states.

An input to an AGS is a guarded string $\alpha_{0} p_{1} \alpha_{1} p_{2} \alpha_{2} \cdots p_{n} \alpha_{n}$. Intuitively, it operates as follows. It starts with a pebble on a nondeterministically chosen start state and its input head scanning $\alpha_{0}$. In the course of the computation, say the pebble is occupying state $s$ and the input head is scanning $\alpha_{i}$. If $i<n$, it may read the next action symbol $p_{i+1}$ from the input string and move the pebble to any nondeterministically chosen state $t$ such that there is an action transition from $s$ to $t$ with label $p_{i+1}$. The input head is advanced past $p_{i+1}$ in the input string and is now scanning $\alpha_{i+1}$. Also while scanning $\alpha_{i}$, it may slide the pebble along an enabled test transition at any time without advancing the input head. A test transition is enabled if $\alpha_{i} \leq b$, where $b$ is the label of the transition. The automaton accepts if it is ever scanning $\alpha_{n}$ while the pebble is on an accept state. Thus the automaton accepts a guarded string $x$ if there is a directed path $\pi$ from start to accept such that $x \leq e$, where $e$ is the product of the labels of the edges along $\pi$.

Formally, a (nondeterministic) automaton on guarded strings (AGS) over $\Sigma$ and $T$ is a tuple

$$
M=(Q, \Delta, \text { start, accept }),
$$

where $Q$ is a set of states, start $\subseteq Q$ are the start states, accept $\subseteq Q$ are the accept states, and $\Delta$ is the transition function

$$
\Delta:(\Sigma+\mathrm{At}) \rightarrow Q \rightarrow 2^{Q},
$$

where + denotes disjoint (marked) union.

The definition of acceptance involves the Kleisli composition $\bullet$ and asterate $\dagger$ operations on maps $Q \rightarrow 2^{Q}$ defined by:

$$
\begin{array}{rlr}
R \bullet S \stackrel{\text { def }}{=} s \mapsto \bigcup_{t \in S(s)} R(t) & R^{0} \stackrel{\text { def }}{=} s \mapsto\{s\} \\
R^{\dagger} \stackrel{\text { def }}{=} \bigcup_{n \geq 0} R^{n} & R^{n+1} \stackrel{\text { def }}{=} R \bullet R^{n} .
\end{array}
$$

The map $\Delta$ generates a map 


$$
\hat{\Delta}:(\Sigma+\text { At }) \rightarrow Q \rightarrow 2^{Q} \quad \hat{\Delta}_{\alpha} \stackrel{\text { def }}{=} \Delta_{\alpha}^{\dagger} \quad \hat{\Delta}_{p} \stackrel{\text { def }}{=} \Delta_{p} .
$$

Intuitively, $\hat{\Delta}_{\alpha}(s)$ accumulates all states accessible from $s$ by a sequence of test transitions enabled under $\alpha$. The map $\hat{\Delta}$ extends further to a monoid homomorphism

$$
\hat{\Delta}:(\Sigma+\mathrm{At})^{*} \rightarrow Q \rightarrow 2^{Q} \quad \hat{\Delta}_{\varepsilon} \stackrel{\text { def }}{=} s \mapsto\{s\} \quad \hat{\Delta}_{x y} \stackrel{\text { def }}{=} \hat{\Delta}_{y} \bullet \hat{\Delta}_{x}
$$

from the free monoid $(\Sigma+\mathrm{At})^{*}$ to the monoid $Q \rightarrow 2^{Q}$ under Kleisli composition. The guarded strings GS $=$ At $\cdot(\Sigma \cdot A t)^{*}$ form a submonoid of $(\Sigma+A t)^{*}$. The automaton $M$ accepts $x \in$ GS if there exist $s \in$ start and $t \in$ accept such that $t \in \hat{\Delta}_{x}(s)$. The set of guarded strings accepted by $M$ is denoted GS $(M)$.

\subsubsection{Deterministic Automata}

The definition of deterministic AGS here differs from that of Kozen (2003) so as to conform to the coalgebraic structure to be introduced in Sect. 15.4, but the difference is inessential. In Kozen (2003) the set of states of a deterministic AGS is separated into disjoint sets of action states and test states, whereas here we have elided the action states.

A deterministic automaton on guarded strings (AGS) over $\Sigma$ and $T$ is a structure

$$
M=(Q, \delta, \varepsilon, \text { start })
$$

where $Q$ is a set of states, start $\in Q$ is the start state, and

$$
\delta: \text { At } \cdot \Sigma \rightarrow Q \rightarrow Q \quad \varepsilon: \text { At } \rightarrow Q \rightarrow 2
$$

with components

$$
\delta_{\alpha p}: Q \rightarrow Q \quad \varepsilon_{\alpha}: Q \rightarrow 2
$$

for $\alpha \in$ At and $p \in \Sigma$. The components $\varepsilon_{\alpha}$ play the same role as the accept states in a nondeterministic automaton.

Define the function $L: Q \rightarrow \mathrm{GS} \rightarrow 2$ inductively as follows:

$$
L(u)(\alpha) \stackrel{\text { def }}{=} \varepsilon_{\alpha}(u) \quad L(u)(\alpha p y) \stackrel{\text { def }}{=} L\left(\delta_{\alpha p}(u)\right)(y),
$$

where $y \in \mathrm{GS}, \alpha \in \mathrm{At}$, and $p \in \Sigma$. The machine is said to accept $x \in \mathrm{GS}$ if $L($ start $)(x)=1$. The set of guarded strings accepted by $M$ is denoted $\mathrm{GS}(M)$. Identifying a subset of GS with its characteristic function GS $\rightarrow 2$, we can write $\mathrm{GS}(M)=L($ start). 
The map $\delta$ extends to a monoid homomorphism $\hat{\delta}:(\text { At } \cdot \Sigma)^{*} \rightarrow Q \rightarrow Q$ from the free monoid $(\text { At } \cdot \Sigma)^{*}$ to the monoid $Q \rightarrow Q$ under functional composition.

\subsubsection{Determinization}

Determinization is effected by a subset construction similar to that for ordinary automata. Given a nondeterministic AGS

$$
N=(Q, \Delta, \text { start, accept })
$$

there is an equivalent deterministic AGS

$$
M=\left(2^{Q}, \delta, \varepsilon, \text { start }\right)
$$

where for $A \subseteq Q$,

$$
\varepsilon_{\alpha}(A) \stackrel{\text { def }}{=} \begin{cases}1, & \text { if } \exists s \in A \exists t \in \text { accept } t \in \hat{\Delta}_{\alpha}(s), \quad \delta_{\alpha p}(A) \stackrel{\text { def }}{=} \bigcup_{s \in A} \hat{\Delta}_{\alpha p}(s) . \\ 0, & \text { otherwise }\end{cases}
$$

One can show by a straightforward induction on the length of $x \in$ GS that for all $A \subseteq Q$

$$
L(A)(x)= \begin{cases}1, & \text { if } \exists s \in A \exists t \in \text { accept } t \in \hat{\Delta}_{x}(s) \\ 0, & \text { otherwise }\end{cases}
$$

in particular,

$$
L(\operatorname{start})(x)=1 \Leftrightarrow \exists s \in \operatorname{start} \exists t \in \text { accept } t \in \hat{\Delta}_{x}(s) .
$$

As these are exactly the acceptance criteria for $M$ and $N$ respectively, the two machines accept the same set of guarded strings.

\subsection{Kleene Coalgebra with Tests (KCT)}

A Kleene coalgebra with tests $(\mathrm{KCT})$ is very much like Kleene coalgebra (KC) (Rutten 1998), but with the addition of Boolean tests. Formally, a Kleene coalgebra with tests (KCT) over $\Sigma$ and $T$ is a structure

$$
M=(Q, \delta, \varepsilon)
$$


where $Q$ is a set of states and

$$
\delta: \text { At } \cdot \Sigma \rightarrow Q \rightarrow Q \quad \varepsilon: \text { At } \rightarrow Q \rightarrow 2
$$

for $\alpha \in$ At and $p \in \Sigma$, exactly as in deterministic automata on guarded strings. Thus we can view a KCT as simply a deterministic AGS without a designated start state.

A KCT morphism $h:(Q, \delta, \varepsilon) \rightarrow\left(Q^{\prime}, \delta^{\prime}, \varepsilon^{\prime}\right)$ is a set map $h: Q \rightarrow Q^{\prime}$ that commutes with $\delta, \delta^{\prime}$ and $\varepsilon, \varepsilon^{\prime}$; that is,

$$
\delta_{\alpha p}^{\prime}(h(u))=h\left(\delta_{\alpha p}(u)\right) \quad \varepsilon_{\alpha}^{\prime}(h(u))=\varepsilon_{\alpha}(u) .
$$

We denote the category of KCTs and KCT morphisms over $\Sigma$ and $T$ also by KCT.

\subsubsection{The Brzozowski Derivative, Semantic Form}

There is a natural KCT over $\Sigma$ and $T$ defined in terms of the Brzozowski derivative on sets of guarded strings. The traditional Brzozowski derivative (Brzozowski 1964) is a kind of residuation operator on sets of ordinary strings. The current form is quite similar, except that we extend the definition to accommodate tests.

We define two maps

$$
D: \text { At } \cdot \Sigma \rightarrow 2^{\mathrm{GS}} \rightarrow 2^{\mathrm{GS}} \quad E: \text { At } \rightarrow 2^{\mathrm{GS}} \rightarrow 2,
$$

where for $R \subseteq \mathrm{GS}$,

$$
D_{\alpha p}(R) \stackrel{\text { def }}{=}\{y \in \mathrm{GS} \mid \alpha p y \in R\} \quad E_{\alpha}(R) \stackrel{\text { def }}{=} \begin{cases}1, & \text { if } \alpha \in R, \\ 0, & \text { if } \alpha \notin R .\end{cases}
$$

It is clear that the structure

$$
\operatorname{Brz} \stackrel{\text { def }}{=}\left(2^{\mathrm{GS}}, D, E\right)
$$

forms a KCT. Indeed, it is the final object in the category KCT: for any KCT $M=$ $(Q, \delta, \varepsilon)$, the function $L: Q \rightarrow 2^{\mathrm{GS}}$ defined in (15.1) is the unique KCT morphism $L: M \rightarrow \mathrm{Brz}$.

\subsubsection{The Brzozowski Derivative, Syntactic Form}

As with Brzozowski's original formulation (Brzozowski 1964), there is also a syntactic form of the Brzozowski derivative defined on KAT expressions. Let Exp denote 
the set of KAT expressions over $\Sigma$ and $T$. We define a family of derivative operators

$$
D: \text { At } \cdot \Sigma \rightarrow \operatorname{Exp} \rightarrow \operatorname{Exp} \quad E: \text { At } \rightarrow \operatorname{Exp} \rightarrow 2
$$

consisting of components

$$
D_{\alpha p}: \operatorname{Exp} \rightarrow \operatorname{Exp} \quad E_{\alpha}: \operatorname{Exp} \rightarrow 2
$$

defined inductively as follows. For $\alpha \in$ At, $p, q \in \Sigma$, and $b \in B$,

$$
\begin{aligned}
& \begin{aligned}
D_{\alpha p}\left(e_{1}+e_{2}\right) & \stackrel{\text { def }}{=} D_{\alpha p}\left(e_{1}\right)+D_{\alpha p}\left(e_{2}\right) \\
D_{\alpha p}\left(e_{1} e_{2}\right) & \stackrel{\text { def }}{=} D_{\alpha p}\left(e_{1}\right) \cdot e_{2}+E_{\alpha}\left(e_{1}\right) \cdot D_{\alpha p}\left(e_{2}\right)
\end{aligned} D_{\alpha p}(q) \stackrel{\text { def }}{=} \begin{cases}1, & \text { if } p=q, \\
0, & \text { otherwise, }\end{cases} \\
& D_{\alpha p}\left(e^{*}\right) \stackrel{\text { def }}{=} D_{\alpha p}(e) \cdot e^{*} \quad D_{\alpha p}(b) \stackrel{\text { def }}{=} 0 \text {. }
\end{aligned}
$$

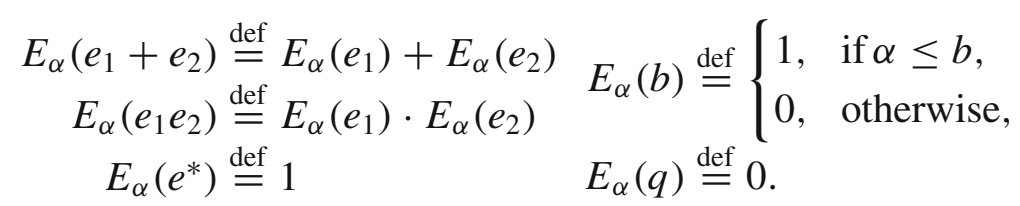

These operators on KAT expressions are collectively called the syntactic Brzozowski derivative.

The map $E_{\alpha}$ is just the evaluation morphism that for any KAT expression substitutes 0 for any $p \in \Sigma, 1$ for any $b \in T$ such that $\alpha \leq b$, and 0 for any $b \in T$ such that $\alpha \leq \bar{b}$, then simplifies the resulting expression over the two-element Kleene algebra 2. It is easily shown that for any KAT expression $e$,

$$
E_{\alpha}(e)=\left\{\begin{array}{ll}
1, & \text { if } \alpha \leq e, \\
0, & \text { if } \alpha \not \leq e
\end{array}= \begin{cases}1, & \text { if } \alpha \in \mathrm{GS}(e), \\
0, & \text { if } \alpha \notin \mathrm{GS}(e) .\end{cases}\right.
$$

The structure $(\operatorname{Exp}, D, E)$ is a KCT in the sense of Sect. 15.4, thus there is a unique KCT morphism $L:$ Exp $\rightarrow$ Brz to the final coalgebra Brz defined in (15.1). We will show that $L(e)=\mathrm{GS}(e)$, where GS is the traditional interpretation of KAT expressions mentioned in Sect. 15.2.2.

Lemma 15.4.1 For all $\alpha \in \mathrm{At}, p \in \Sigma$, and $e, e^{\prime} \in \mathrm{Exp}$,

$$
\alpha p e^{\prime} \leq e \Leftrightarrow e^{\prime} \leq D_{\alpha p}(e)
$$

Proof For the forward implication,

$$
D_{\alpha p}\left(\alpha p e^{\prime}\right)=D_{\alpha p}(\alpha) p e^{\prime}+E_{\alpha}(\alpha) D_{\alpha p}(p) e^{\prime}+E_{\alpha}(\alpha) E_{\alpha}(p) D_{\alpha p}\left(e^{\prime}\right)=e^{\prime} .
$$

By monotonicity of $D_{\alpha p}$, 


$$
\alpha p e^{\prime} \leq e \Rightarrow e^{\prime}=D_{\alpha p}\left(\alpha p e^{\prime}\right) \leq D_{\alpha p}(e)
$$

For the reverse implication, it suffices to show $\alpha p D_{\alpha p}(e) \leq e$. We proceed by induction on the structure of $e$. For $p \in \Sigma$,

$$
\alpha p D_{\alpha p}(p)=\alpha p \leq p
$$

For the case $e_{1} e_{2}$,

$$
\begin{aligned}
\alpha p D_{\alpha p}\left(e_{1} e_{2}\right) & =\alpha p D_{\alpha p}\left(e_{1}\right) e_{2}+\alpha p E_{\alpha}\left(e_{1}\right) D_{\alpha p}\left(e_{2}\right) \\
& =\alpha p D_{\alpha p}\left(e_{1}\right) e_{2}+\alpha E_{\alpha}\left(e_{1}\right) \alpha p D_{\alpha p}\left(e_{2}\right) \\
& \leq e_{1} e_{2} .
\end{aligned}
$$

For the case $e^{*}$,

$$
\alpha p D_{\alpha p}\left(e^{*}\right)=\alpha p D_{\alpha p}(e) e^{*} \leq e e^{*} \leq e^{*}
$$

All other cases are equally straightforward.

Theorem 15.4.2 For all KAT expressions $e$, GS $(e)=L(e)$. Thus the set accepted by the automaton (Exp, D, E,e) is $\mathrm{GS}(e)$.

Proof We wish to show that for all $x \in \mathrm{GS}, x \in \mathrm{GS}(e)$ iff $L(e)(x)=1$. By the completeness theorem for KAT (Kozen and Smith 1996), we have $x \in \mathrm{GS}(e)$ iff $x \leq e$, so it suffices to show that $x \leq e$ iff $L(e)(x)=1$. We proceed by induction on the length of $x$. The basis for $x$ an atom $\alpha$ is immediate from the definition of $E_{\alpha}$. For $x=\alpha p y$, by Lemma 15.4.1,

$$
\alpha p y \leq e \Leftrightarrow y \leq D_{\alpha p}(e) \Leftrightarrow L\left(D_{\alpha p}(e)\right)(y)=1 \Leftrightarrow L(e)(\text { apy })=1 .
$$

\subsection{Completeness}

\subsubsection{Bisimulation on KCTs}

A bisimulation between two $\mathrm{KCT}_{\mathrm{s}} M=(Q, \delta, \varepsilon)$ and $M^{\prime}=\left(Q^{\prime}, \delta^{\prime}, \varepsilon^{\prime}\right)$ is a binary relation $\equiv$ between $Q$ and $Q^{\prime}$ such that if $s \in Q, t \in Q^{\prime}$, and $s \equiv t$, then for all $\alpha \in$ At and $p \in \Sigma$,

(i) $\varepsilon_{\alpha}(s)=\varepsilon_{\alpha}^{\prime}(t)$; and

(ii) $\delta_{\alpha p}(s) \equiv \delta_{\alpha p}^{\prime}(t)$. 
Lemma 15.5.1 The relation

$$
s \hat{\equiv} t \stackrel{\text { def }}{\Longleftrightarrow} L(s)=L(t)
$$

is the unique maximal bisimulation between $M$ and $M^{\prime}$.

Proof It is easily shown that $\hat{\equiv}$ satisfies (i) and (ii). Moreover, if $\equiv$ is any relation satisfying (i) and (ii), one can show by a straightforward inductive argument that $\equiv$ refines $\hat{\equiv}$, thus $\hat{\equiv}$ is the unique maximal relation satisfying (i) and (ii).

An autobisimulation is a bisimulation between $M$ and itself. Bisimulations are closed under relational composition and arbitrary union, and the identity relation is an autobisimulation. Thus the reflexive, symmetric, and transitive closure of an autobisimulation is again an autobisimulation. An autobisimulation that is so closed is called a $\mathrm{KCT}$-congruence. $\mathrm{KCT}$-congruences are exactly the kernels of KCTmorphisms.

A KCT is bisimilar to its quotient by any KCT-congruence under the map $\{(s,[s]) \mid s \in Q\}$, where $[s]$ is the KCT-congruence class of $s$. The quotient by the unique maximal autobisimulation is a sub-coalgebra of $\mathrm{Brz}$, the final coalgebra.

\subsubsection{Bisimulation on Deterministic Automata}

For deterministic automata, we add an extra condition. A bisimulation between two deterministic AGS $M=(Q, \delta, \varepsilon$, start $)$ and $M^{\prime}=\left(Q^{\prime}, \delta^{\prime}, \varepsilon^{\prime}\right.$, start' $)$ is a bisimulation $\equiv$ between the underlying $\operatorname{KCTs}(Q, \delta, \varepsilon)$ and $\left(Q^{\prime}, \delta^{\prime}, \varepsilon^{\prime}\right)$ such that start $\equiv$ start'. Two automata are bisimilar if there exists a bisimulation between them.

Lemma 15.5.2 $M$ and $M^{\prime}$ are bisimilar iff $\mathrm{GS}(M)=\mathrm{GS}\left(M^{\prime}\right)$.

Proof Let $\hat{\equiv}$ be the relation defined in the proof of Lemma 15.5.1. If $\mathrm{GS}(M)=$ $\mathrm{GS}\left(M^{\prime}\right)$, then $L($ start $)=L\left(\right.$ start $\left.^{\prime}\right)$ by the definition of acceptance, therefore start $\hat{\equiv}$ start'. Then $M$ and $M^{\prime}$ are bisimilar under $\hat{\equiv}$.

Conversely, if there exists a bisimulation $\equiv$ between $M$ and $M^{\prime}$, then start $\equiv$ start', and by Lemma 15.5.1, 三 refines $\hat{\equiv}$, therefore start $\hat{\equiv}$ start'. Thus $\hat{\equiv}$ is a bisimulation of automata.

The quotient of an automaton by its unique maximal autobisimulation gives the unique minimal equivalent automaton (ignoring inaccessible states).

Theorem 15.5.3 (Completeness) The following are equivalent:

(i) the automata $(\operatorname{Exp}, D, E, e)$ and $\left(\operatorname{Exp}, D, E, e^{\prime}\right)$ are bisimilar;

(ii) $L(e)=L\left(e^{\prime}\right)$;

(iii) $\mathrm{GS}(e)=\mathrm{GS}\left(e^{\prime}\right)$; 
(iv) $e$ and $e^{\prime}$ are equivalent modulo the axioms of KAT.

Proof The equivalence of (i)-(iii) follows from Theorem 15.4.2 and Lemma 15.5.2. The equivalence of (iii) and (iv) are just the soundness and completeness of KAT for the guarded string model (Kozen and Smith 1996).

\subsection{Complexity}

Let $\operatorname{Exp}_{e}$ denote the subautomaton of (Exp, $\left.D, E, e\right)$ consisting of those expressions that are accessible from $e$; that is, those expressions of the form $\hat{D}_{x}(e)$ for some $x \in(\text { At } \cdot \Sigma)^{*}$. Theorem 15.5 .3 by itself is not very useful as a deductive system or decision procedure for equivalence, because $\operatorname{Exp}_{e}$ is not a finite system in general. However, equivalent finite systems exist. In particular, by Theorem 15.5.3, KAT equivalence is the maximal congruence on Exp. The quotient with respect to this relation, ignoring inaccessible states, gives the minimal deterministic AGS accepting $\mathrm{GS}(e)$, which is finite since $\mathrm{GS}(e)$ is regular.

Unfortunately, to construct this automaton directly, we would need an independent algorithm to decide KAT equivalence. However, we can obtain finite automata with finer congruences that are easier to decide than full KAT equivalence. Chen and Pucella (2003) use equivalence modulo additive associativity, commutativity, and idempotence (ACI-equivalence). Here we consider equivalence modulo the axioms of idempotent commutative monoids for,+ 0 and the axioms

$$
1 \cdot x=x \quad 0 \cdot x=0 \quad(x+y) \cdot z=x z+y z .
$$

Multiplicative associativity is not assumed, nor is left distributivity. We might call structures satisfying these axioms right presemirings. We denote by $\approx$ the KATcongruence on terms generated by these axioms. We will show that $\operatorname{Exp}_{e} / \approx$ has finitely many accessible classes. It is a coarser relation than ACI-equivalence, therefore has fewer classes, but is still easy to decide, as there are normal forms up to additive commutativity. Of course, it makes the most sense to use the coarsest relation possible that is easily decidable, because coarser relations give smaller automata.

Because there are only finitely many $\approx$-classes accessible from $e$, the quotient automaton $\operatorname{Exp}_{e} / \approx$ is finite, and we can use it to obtain finite coinductive equivalence proofs. More interestingly, we will also show that $\operatorname{Exp}_{e} / \approx$ is a homomorphic image of a deterministic automaton $M_{e}$ obtained by creating a nondeterministic AGS $N_{e}$ from the expression $e$ by a Kleene construction, then determinizing $N_{e}$ by a subset construction as described in Sect. 15.3.4. This characterization gives a bound on the size of $\operatorname{Exp}_{e} / \approx$, which we can then use to argue that coinductive equivalence proofs can be generated automatically in PSPACE.

Lemma 15.6.1 The relation $\approx$ is a $\mathrm{KCT}$-congruence on Exp. 
Proof We must show that if $e \approx e^{\prime}$, then $E_{\alpha}(e)=E_{\alpha}\left(e^{\prime}\right)$ and $D_{\alpha p}(e) \approx D_{\alpha p}\left(e^{\prime}\right)$. The first conclusion follows from Theorem 15.5.3 and the fact that $\approx$ refines KATequivalence.

For the additive axioms of idempotent commutative monoids, the second conclusion follows from the additivity of $D_{\alpha p}$.

For the axioms (15.2),

$$
\begin{aligned}
D_{\alpha p}(1 x) & =D_{\alpha p}(1) x+E_{\alpha}(1) D_{\alpha p}(x)=0 x+1 D_{\alpha p}(x) \approx D_{\alpha p}(x) \\
D_{\alpha p}(0 x) & =D_{\alpha p}(0) x+E_{\alpha}(0) D_{\alpha p}(x)=0 x+0 D_{\alpha p}(x) \approx D_{\alpha p}(0) \\
D_{\alpha p}((x+y) z) & =\left(D_{\alpha p}(x)+D_{\alpha p}(y)\right) z+\left(E_{\alpha}(x)+E_{\alpha}(y)\right) D_{\alpha p}(z) \\
& \approx D_{\alpha p}(x) z+E_{\alpha}(x) D_{\alpha p}(z)+D_{\alpha p}(y) z+E_{\alpha}(y) D_{\alpha p}(z) \\
& =D_{\alpha p}(x z+y z) .
\end{aligned}
$$

Finally, we must show that if $e_{1} \approx e_{2}$, then $D_{\alpha p}\left(e_{1}+e_{3}\right) \approx D_{\alpha p}\left(e_{2}+e_{3}\right), D_{\alpha p}\left(e_{1} e_{3}\right)$ $\approx D_{\alpha p}\left(e_{2} e_{3}\right), D_{\alpha p}\left(e_{3} e_{1}\right) \approx D_{\alpha p}\left(e_{3} e_{2}\right)$, and $D_{\alpha p}\left(e_{1}^{*}\right) \approx D_{\alpha p}\left(e_{2}^{*}\right)$. These arguments are all quite easy. For example,

$$
\begin{aligned}
D_{\alpha p}\left(e_{1} e_{3}\right) & =D_{\alpha p}\left(e_{1}\right) e_{3}+E_{\alpha}\left(e_{1}\right) D_{\alpha p}\left(e_{3}\right) \\
& \approx D_{\alpha p}\left(e_{2}\right) e_{3}+E_{\alpha}\left(e_{2}\right) D_{\alpha p}\left(e_{3}\right)=D_{\alpha p}\left(e_{2} e_{3}\right)
\end{aligned}
$$

and

$$
D_{\alpha p}\left(e_{1}^{*}\right)=D_{\alpha p}\left(e_{1}\right) e_{1}^{*} \approx D_{\alpha p}\left(e_{2}\right) e_{2}^{*}=D_{\alpha p}\left(e_{2}^{*}\right)
$$

\subsubsection{Closure}

To establish the finiteness of the quotient automaton $\operatorname{Exp}_{e} / \approx$ and explain its relationship to the Kleene construction, we derive a formal relationship between the set of accessible $\approx$-classes of derivatives $\left\{\hat{D}_{x}(e) / \approx \mid x \in(\text { At } \cdot \Sigma)^{*}\right\}$ and certain sets of terms derived from $e$.

For KAT term $e$, we define the closure of $e$, denoted $\mathrm{cl}(e)$, to be the smallest set of terms containing $e$ and 1 and closed under the following rules:

$$
\begin{array}{lcc}
\frac{e \in \operatorname{cl}\left(e_{1}\right)}{e \in \operatorname{cl}\left(e_{1}+e_{2}\right)} & \frac{e \in \operatorname{cl}\left(e_{1}\right)}{e e_{2} \in \operatorname{cl}\left(e_{1} e_{2}\right)} & \frac{e \in \operatorname{cl}\left(e_{1}\right)}{e e_{1}^{*} \in \operatorname{cl}\left(e_{1}^{*}\right)} \\
\frac{e \in \operatorname{cl}\left(e_{2}\right)}{e \in \operatorname{cl}\left(e_{1}+e_{2}\right)} & \frac{e \in \operatorname{cl}\left(e_{2}\right)}{e \in \operatorname{cl}\left(e_{1} e_{2}\right)} & \frac{e \in \operatorname{cl}(b)}{e \in \operatorname{cl}(\bar{b})}
\end{array}
$$


Lemma 15.6.2 The set $\mathrm{cl}(e)$ contains at most $|e|+1$ elements, where $|e|$ is the number of subterms of $e$.

Proof We show by induction on $e$ that $\mathrm{cl}^{\prime}(e)$ contains at most $|e|$ elements, where $\operatorname{cl}^{\prime}(e)=\operatorname{cl}(e)-\{1\}$. For $e \in \Sigma \cup T, \operatorname{cl}^{\prime}(e)=\{e\}$. For the other operators, from the rules (15.3) we have

$$
\begin{aligned}
\operatorname{cl}^{\prime}(\bar{b}) & =\{\bar{b}\} \cup \mathrm{cl}^{\prime}(b), \\
\operatorname{cl}^{\prime}\left(e_{1}+e_{2}\right) & =\left\{e_{1}+e_{2}\right\} \cup \operatorname{cl}^{\prime}\left(e_{1}\right) \cup \operatorname{cl}^{\prime}\left(e_{2}\right), \\
\operatorname{cl}^{\prime}\left(e_{1} e_{2}\right) & =\left\{e_{1} e_{2}\right\} \cup\left\{e e_{2} \mid e \in \operatorname{cl}^{\prime}\left(e_{1}\right)\right\} \cup \operatorname{cl}^{\prime}\left(e_{2}\right), \\
\operatorname{cl}^{\prime}\left(e_{1}^{*}\right) & =\left\{e_{1}^{*}\right\} \cup\left\{e e_{1}^{*} \mid e \in \operatorname{cl}^{\prime}\left(e_{1}\right)\right\} .
\end{aligned}
$$

The result follows.

\subsubsection{Set Representation of Derivatives}

We now construct a nondeterministic transition function $\Delta$ on the set of states Exp + $($ At $\times$ Exp) as follows. The elements of Exp are called test states and the elements of At $\times$ Exp are called action states. The test transitions go only from test states to action states, and the action transitions go only from action states to test states. Thus for $\alpha \in$ At and $p \in \Sigma$,

$$
\Delta_{\alpha}: \operatorname{Exp} \rightarrow 2^{\text {At } \times \operatorname{Exp}} \quad \Delta_{p}: \text { At } \times \operatorname{Exp} \rightarrow 2^{\operatorname{Exp} .}
$$

The test transitions are deterministic: $\Delta_{\alpha}(e) \stackrel{\text { def }}{=}\{(\alpha, e)\}$. The action transitions are defined inductively:

$$
\begin{gathered}
\Delta_{p}(\alpha, q) \stackrel{\text { def }}{=} \begin{cases}\{1\}, & \text { if } q \in \Sigma \text { and } q=p, \\
\emptyset, & \text { if } q \in \Sigma \text { and } q \neq p \text { or } q \in B,\end{cases} \\
\Delta_{p}\left(\alpha, e_{1}+e_{2}\right) \stackrel{\text { def }}{=} \Delta_{p}\left(\alpha, e_{1}\right) \cup \Delta_{p}\left(\alpha, e_{2}\right), \\
\Delta_{p}\left(\alpha, e_{1} e_{2}\right) \stackrel{\text { def }}{=} \begin{cases}\left\{e e_{2} \mid e \in \Delta_{p}\left(\alpha, e_{1}\right)\right\} \cup \Delta_{p}\left(\alpha, e_{2}\right), & \text { if } E_{\alpha}\left(e_{1}\right)=1, \\
\left\{e e_{2} \mid e \in \Delta_{p}\left(\alpha, e_{1}\right)\right\}, & \text { if } E_{\alpha}\left(e_{1}\right)=0,\end{cases} \\
\Delta_{p}\left(\alpha, e_{1}^{*}\right) \stackrel{\text { def }}{=}\left\{e e_{1}^{*} \mid e \in \Delta_{p}\left(\alpha, e_{1}\right)\right\} .
\end{gathered}
$$

Due to the bipartite structure of the states, we have $\hat{\Delta}_{\alpha p}=\Delta_{p} \bullet \Delta_{\alpha}$, where $\hat{\Delta}$ is the extension of $\Delta$ defined in Sect. 15.3.2. Then

$$
\hat{\Delta}_{\alpha p}(e)=\left(\Delta_{p} \bullet \Delta_{\alpha}\right)(e)=\bigcup\left\{\Delta_{p}(\alpha, e)\right\}=\Delta_{p}(\alpha, e) .
$$


We thus have

$$
\begin{gathered}
\hat{\Delta}_{\alpha p}(q) \stackrel{\text { def }}{=} \begin{cases}\{1\}, & \text { if } q \in \Sigma \text { and } q=p, \\
\emptyset, & \text { if } q \in \Sigma \text { and } q \neq p \text { or } q \in B,\end{cases} \\
\hat{\Delta}_{\alpha p}\left(e_{1}+e_{2}\right) \stackrel{\text { def }}{=} \hat{\Delta}_{\alpha p}\left(e_{1}\right) \cup \hat{\Delta}_{\alpha p}\left(e_{2}\right), \\
\hat{\Delta}_{\alpha p}\left(e_{1} e_{2}\right) \stackrel{\text { def }}{=} \begin{cases}\left\{e e_{2} \mid e \in \hat{\Delta}_{\alpha p}\left(e_{1}\right)\right\} \cup \hat{\Delta}_{\alpha p}\left(e_{2}\right), & \text { if } E_{\alpha}\left(e_{1}\right)=1, \\
\left\{e e_{2} \mid e \in \hat{\Delta}_{\alpha p}\left(e_{1}\right)\right\}, & \text { if } E_{\alpha}\left(e_{1}\right)=0,\end{cases} \\
\hat{\Delta}_{\alpha p}\left(e_{1}^{*}\right) \stackrel{\text { def }}{=}\left\{e e_{1}^{*} \mid e \in \hat{\Delta}_{\alpha p}\left(e_{1}\right)\right\} .
\end{gathered}
$$

Lemma 15.6.3 For all KAT terms $e$ and $x \in(\mathrm{At} \cdot \Sigma)^{*}, \hat{\Delta}_{x}(e) \subseteq \operatorname{cl}(e)$.

Proof We first show that for $\alpha \in$ At and $p \in \Sigma, \hat{\Delta}_{\alpha p}(e) \subseteq \operatorname{cl}(e)$ by induction on the structure of $e$. The cases $e \in \Sigma$ or $e \in B$ are easy. For the other operators,

$$
\begin{aligned}
& \hat{\Delta}_{\alpha p}\left(e_{1}+e_{2}\right)=\hat{\Delta}_{\alpha p}\left(e_{1}\right) \cup \hat{\Delta}_{\alpha p}\left(e_{2}\right) \subseteq \operatorname{cl}\left(e_{1}\right) \cup \operatorname{cl}\left(e_{2}\right) \subseteq \operatorname{cl}\left(e_{1}+e_{2}\right) \\
& \hat{\Delta}_{\alpha p}\left(e_{1} e_{2}\right)= \begin{cases}\left\{e e_{2} \mid e \in \hat{\Delta}_{\alpha p}\left(e_{1}\right)\right\} \cup \hat{\Delta}_{\alpha p}\left(e_{2}\right), & \text { if } E_{\alpha}\left(e_{1}\right)=1 \\
\left\{e e_{2} \mid e \in \hat{\Delta}_{\alpha p}\left(e_{1}\right)\right\}, & \text { if } E_{\alpha}\left(e_{1}\right)=0\end{cases} \\
& \subseteq\left\{e e_{2} \mid e \in \operatorname{cl}\left(e_{1}\right)\right\} \cup \operatorname{cl}\left(e_{2}\right) \subseteq \operatorname{cl}\left(e_{1} e_{2}\right) \\
& \hat{\Delta}_{\alpha p}\left(e_{1}^{*}\right)=\left\{e e_{1}^{*} \mid e \in \hat{\Delta}_{\alpha p}\left(e_{1}\right)\right\} \subseteq\left\{e e_{1}^{*} \mid e \in \operatorname{cl}\left(e_{1}\right)\right\} \subseteq \operatorname{cl}\left(e_{1}^{*}\right) \text {. }
\end{aligned}
$$

For arbitrary $x \in(\mathrm{At} \cdot \Sigma)^{*}$, we proceed by induction on the length of $x$. The base case $x=\varepsilon$ is easy and the case $x=\alpha p$ is given by the previous argument. For $x \neq \varepsilon$ and $y \neq \varepsilon$,

$$
\begin{aligned}
\hat{\Delta}_{x y}(e) & =\left(\hat{\Delta}_{y} \bullet \hat{\Delta}_{x}\right)(e)=\bigcup\left\{\hat{\Delta}_{y}(d) \mid d \in \hat{\Delta}_{x}(e)\right\} \\
& \subseteq \bigcup\{\operatorname{cl}(d) \mid d \in \operatorname{cl}(e)\}=\operatorname{cl}(e)
\end{aligned}
$$

Lemma 15.6.4 For all KAT terms $e$ and $x \in(\mathrm{At} \cdot \Sigma)^{*}, \hat{D}_{x}(e) \approx \sum \hat{\Delta}_{x}(e)$.

Proof We first show that for $\alpha \in$ At and $p \in \Sigma, D_{\alpha p}(e) \approx \sum \hat{\Delta}_{\alpha p}(e)$ by induction on the structure of $e$. For $q \in \Sigma$, we have

$$
D_{\alpha p}(q)=\left\{\begin{array}{ll}
1, & \text { if } p=q \\
0, & \text { if } p \neq q
\end{array}=\left\{\begin{array}{ll}
\sum\{1\}, & \text { if } p=q \\
\sum \emptyset, & \text { if } p \neq q
\end{array}=\sum \hat{\Delta}_{\alpha p}(q)\right.\right.
$$

For $b \in B$,

$$
D_{\alpha p}(b)=0=\sum \emptyset=\sum \hat{\Delta}_{\alpha p}(b)
$$


For the other operators,

$$
\begin{aligned}
& D_{\alpha p}\left(e_{1}+e_{2}\right)=D_{\alpha p}\left(e_{1}\right)+D_{\alpha p}\left(e_{2}\right) \approx \sum \hat{\Delta}_{\alpha p}\left(e_{1}\right)+\sum \hat{\Delta}_{\alpha p}\left(e_{2}\right) \\
& \approx \sum\left(\hat{\Delta}_{\alpha p}\left(e_{1}\right) \cup \hat{\Delta}_{\alpha p}\left(e_{2}\right)\right)=\sum \hat{\Delta}_{\alpha p}\left(e_{1}+e_{2}\right), \\
& D_{\alpha p}\left(e_{1} e_{2}\right)=D_{\alpha p}\left(e_{1}\right) e_{2}+E_{\alpha}\left(e_{1}\right) D_{\alpha p}\left(e_{2}\right) \\
& \approx\left(\sum \hat{\Delta}_{\alpha p}\left(e_{1}\right)\right) e_{2}+E_{\alpha}\left(e_{1}\right) \sum \hat{\Delta}_{\alpha p}\left(e_{2}\right) \\
& \approx \begin{cases}\sum\left\{e e_{2} \mid e \in \hat{\Delta}_{\alpha p}\left(e_{1}\right)\right\}+\sum \hat{\Delta}_{\alpha p}\left(e_{2}\right), & \text { if } E_{\alpha}\left(e_{1}\right)=1 \\
\sum\left\{e e_{2} \mid e \in \hat{\Delta}_{\alpha p}\left(e_{1}\right)\right\}, & \text { if } E_{\alpha}\left(e_{1}\right)=0\end{cases} \\
& \approx \begin{cases}\sum\left(\left\{e e_{2} \mid e \in \hat{\Delta}_{\alpha p}\left(e_{1}\right)\right\} \cup \hat{\Delta}_{\alpha p}\left(e_{2}\right)\right), & \text { if } E_{\alpha}\left(e_{1}\right)=1 \\
\sum\left\{e e_{2} \mid e \in \hat{\Delta}_{\alpha p}\left(e_{1}\right)\right\}, & \text { if } E_{\alpha}\left(e_{1}\right)=0\end{cases} \\
& =\sum \hat{\Delta}_{\alpha p}\left(e_{1} e_{2}\right) \\
& D_{\alpha p}\left(e_{1}^{*}\right)=D_{\alpha p}\left(e_{1}\right) e_{1}^{*} \approx\left(\sum \hat{\Delta}_{\alpha p}\left(e_{1}\right)\right) e_{1}^{*} \\
& \approx \sum\left\{e e_{1}^{*} \mid e \in \hat{\Delta}_{\alpha p}\left(e_{1}\right)\right\}=\sum \hat{\Delta}_{\alpha p}\left(e_{1}^{*}\right)
\end{aligned}
$$

Now we show the result for arbitrary $x \in(\text { At } \cdot \Sigma)^{*}$ by induction on the length of $x$. The case $x=\varepsilon$ is trivial, and the case $x=\alpha p$ is given by the previous argument. Finally, for $x \neq \varepsilon$ and $y \neq \varepsilon$,

$$
\begin{aligned}
\hat{D}_{x y}(e) & =\hat{D}_{y}\left(\hat{D}_{x}(e)\right) \\
& \approx \hat{D}_{y}\left(\sum \hat{\Delta}_{x}(e)\right) \quad \text { by Lemma } 6.1 \\
& =\sum\left\{\hat{D}_{y}(d) \mid d \in \hat{\Delta}_{x}(e)\right\} \\
& \approx \sum\left\{\sum \hat{\Delta}_{y}(d) \mid \in \hat{\Delta}_{x}(e)\right\} \approx \sum \bigcup\left\{\hat{\Delta}_{y}(d) \mid d \in \hat{\Delta}_{x}(e)\right\} \\
& =\sum\left(\hat{\Delta}_{y} \bullet \hat{\Delta}_{x}\right)(e)=\sum \hat{\Delta}_{x y}(e) .
\end{aligned}
$$

Theorem 15.6.5 The automaton $\operatorname{Exp}_{e} / \approx$ has at most $2^{|e|+1}$ accessible states.

Proof The accessible states of $\operatorname{Exp}_{e} / \approx$ are $\left\{\hat{D}_{x}(e) / \approx \mid x \in(\text { At } \cdot \Sigma)^{*}\right\}$, where $d / \approx$ is the congruence class of $d$ modulo $\approx$. The stated bound follows from Lemmas 15.6.2, 15.6.3, and 15.6.4. 


\subsubsection{Brzozowski Meets Kleene}

It is possible to obtain $\operatorname{Exp}_{e} / \approx$ by a Kleene construction to obtain a nondeterministic AGS $N_{e}$ with finitely many states, then apply the construction of Sect. 15.3.4 to obtain a deterministic automaton $M_{e}$ with at most $2^{|e|+1}$ states. The automaton $\operatorname{Exp}_{e} / \approx$ is a homomorphic image of $M_{e}$. A version of Kleene's theorem for KAT terms and automata on guarded strings has been described previously in Kozen (2003), but the current treatment parallels more closely Brzozowski's original treatment for ordinary regular expressions (Brzozowski 1964) and aligns with the general coalgebraic structure of Bonsangue et al. (2007, 2009).

Define the nondeterministic automaton

$$
N_{e} \stackrel{\text { def }}{=}(Q, \Delta, \text { start, accept }),
$$

where the set of states $Q$ is the disjoint union $\operatorname{cl}(e)+(\operatorname{At} \times \operatorname{cl}(e))$, the transition function $\Delta$ is that defined in Sect. 15.6.2, and the start and accept states are

$$
\text { start } \stackrel{\text { def }}{=}\{e\} \quad \text { accept } \stackrel{\text { def }}{=}\left\{(\alpha, d) \mid E_{\alpha}(d)=1\right\} .
$$

That $\Delta_{\alpha} \operatorname{maps} \operatorname{cl}(e)$ to $2^{\operatorname{At} \times \operatorname{cl}(e)}$ is immediate from the definition of $\Delta_{\alpha}$, and that $\Delta_{p}$ maps At $\times \operatorname{cl}(e)$ to $2^{\operatorname{cl}(e)}$ is guaranteed by (15.4) and Lemma 15.6.3.

Now let

$$
M_{e} \stackrel{\text { def }}{=}\left(2^{\text {cl }(e)}, \delta, \varepsilon, \text { start }\right)
$$

be the deterministic automaton obtained from $N_{e}$ by the subset construction as described in Sect. 15.3.4. The start state of $M_{e}$ is $\{e\}$, and $\delta$ and $\varepsilon$ are given by

$$
\delta_{\alpha p}(A)=\bigcup_{d \in A} \hat{\Delta}_{\alpha p}(d) \quad \varepsilon_{\alpha}(A)= \begin{cases}1, & \text { if } \exists d \in A E_{\alpha}(d)=1 \\ 0, & \text { otherwise. }\end{cases}
$$

Note that the accessible states are all of the form $A \subseteq \operatorname{cl}(e)$, thus by Lemma 15.6.2, $M_{e}$ has at most $2^{|e|+1}$ accessible states.

Theorem 15.6.6 For $A \subseteq$ Exp, the map $A \mapsto\left(\sum A\right) / \approx$ is a KCT-morphism. Ignoring inaccessible states, the quotient automaton $\operatorname{Exp}_{e} / \approx$ is the image of $M_{e}$ under this map.

Proof We must show that the function $A \mapsto \sum A$ maps the start state of $M_{e}$ to the start state of $\operatorname{Exp}_{e}$, and that this function is a bisimulation modulo $\approx$. For $\delta$, 


$$
\begin{array}{rlr}
\sum \delta_{\alpha p}(A) & =\sum \bigcup\left\{\hat{\Delta}_{\alpha p}(d) \mid d \in A\right\} & \\
& \approx \sum\left\{\sum \hat{\Delta}_{\alpha p}(d) \mid d \in A\right\} & \\
& \approx \sum\left\{D_{\alpha p}(d) \mid d \in A\right\} & \text { by Lemma } 6.4 \\
& =D_{\alpha p}\left(\sum A\right),
\end{array}
$$

therefore

$$
\left(\sum \delta_{\alpha p}(A)\right) / \approx=\left(D_{\alpha p}\left(\sum A\right)\right) / \approx=D_{\alpha p}\left(\left(\sum A\right) / \approx\right) .
$$

For $\varepsilon$,

$$
\varepsilon_{\alpha}(A)=\left\{\begin{array}{ll}
1, & \text { if } \exists d \in A E_{\alpha}(d)=1 \\
0, & \text { otherwise }
\end{array}=E_{\alpha}\left(\sum A\right)=E_{\alpha}\left(\left(\sum A\right) / \approx\right) .\right.
$$

The map also preserves start states:

$$
\{e\} \mapsto\left(\sum\{e\}\right) / \approx=e / \approx
$$

Thus the map $A \mapsto\left(\sum A\right) / \approx$ is a KCT-morphism mapping $M_{e}$ to $\operatorname{Exp}_{e} / \approx$.

\subsubsection{Automatic Proof Generation in PSPACE}

The results of Sects. 15.6.2 and 15.6.3 give rise to a nondeterministic linear-space algorithm for deciding the equivalence of two given KAT terms. By Savitch's theorem (Savitch 1970), there is a deterministic quadratic-space algorithm. The deterministic algorithm can be used to create bisimulation proofs of equivalence or inequivalence automatically.

To obtain the linear space bound, we first show that each element of $\operatorname{cl}(e)$ corresponds to an occurrence of a subterm of $e$. This lets us use the occurrences of subterms of $e$ as representatives for the elements of $\operatorname{cl}(e)$. To define the correspondence, we view terms as labeled trees; that is, as partial functions

$$
e: \omega^{*} \rightarrow \Sigma \cup T \cup\left\{+, \cdot,^{*},{ }^{-}, 0,1\right\}
$$

with domain of definition dom $e \subseteq \omega^{*}$ such that

- dom $e$ is finite, nonempty, and prefix-closed;

- if $\sigma \in \operatorname{dom} e$ and $e(\sigma)$ is of arity $n$, then $\sigma i \in \operatorname{dom} e$ iff $i<n$. The arities of elements of $\Sigma$ and $T$ are 0 and those of $+, \cdot,^{*},{ }^{-}, 0,1$ are 2, 2, 1, 1, 0, 0 , respectively.

An occurrence of a subterm of $e$ is identified by its position $\sigma \in \operatorname{dom} e$. The subterm at position $\sigma$ is $\lambda \tau . e(\sigma \tau)$, and its domain is $\{\tau \mid \sigma \tau \in \operatorname{dom} e\}$. 
Define a partial function $R: \omega^{*} \times \operatorname{Exp} \rightarrow$ Exp inductively by

$$
\begin{array}{cc}
R\left(0 \sigma, e_{1}+e_{2}\right) \stackrel{\text { def }}{=} R\left(\sigma, e_{1}\right) & R\left(0 \sigma, e_{1} e_{2}\right) \stackrel{\text { def }}{=} R\left(\sigma, e_{1}\right) \cdot e_{2} \\
R\left(1 \sigma, e_{1}+e_{2}\right) \stackrel{\text { def }}{=} R\left(\sigma, e_{2}\right) & R\left(1 \sigma, e_{1} e_{2}\right) \stackrel{\text { def }}{=} R\left(\sigma, e_{2}\right) \\
R\left(0 \sigma, e^{*}\right) \stackrel{\text { def }}{=} R(\sigma, e) \cdot e^{*} & R(\varepsilon, e) \stackrel{\text { def }}{=} e .
\end{array}
$$

One can show by induction that $R(\sigma, e)$ is defined iff $\sigma \in \operatorname{dom} e$, and that a term is in $\operatorname{cl}(e)$ iff it is either 1 or $R(\sigma, e)$ for some $\sigma \in \operatorname{dom} e$.

Now we show how to construct coinductive equivalence and inequivalence proofs for two given terms $e_{1}$ and $e_{2}$. Construct the two nondeterministic AGS $N_{e_{1}}$ and $N_{e_{2}}$ as described in Sect. 15.6.3, representing the states by dom $e_{1}$ and dom $e_{2}$, respectively (assume without loss of generality that $1=R\left(\sigma, e_{1}\right)=R\left(\tau, e_{2}\right)$ for some $\sigma$ and $\left.\tau\right)$. If we like, we can also reduce terms modulo $\approx$, so that if $R\left(\sigma, e_{1}\right) \approx R\left(\tau, e_{1}\right)$, we only need one of $\sigma, \tau$.

Place pebbles on the start states of the two automata. Nondeterministically guess a string $y \in(\text { At } \cdot \Sigma)^{*}$ and move the pebbles to all accessible states according to the transition functions of the two machines. Halt and declare $e_{1}$ and $e_{2}$ inequivalent if there exists $\alpha \in$ At such that

$$
E_{\alpha}\left(\sum_{\tau \in A_{1}} R\left(\tau, e_{1}\right)\right) \neq E_{\alpha}\left(\sum_{\tau \in A_{2}} R\left(\rho, e_{2}\right)\right)
$$

where $A_{1}$ and $A_{2}$ are the sets of states of $N_{e_{1}}$ and $N_{e_{2}}$, respectively, currently occupied by pebbles; we have found a guarded string $x=y \alpha$ accepted by one but not by the other, since

$$
L\left(e_{i}\right)(x)=E_{\alpha}\left(\hat{D}_{y}\left(e_{i}\right)\right)=E_{\alpha}\left(\sum_{\tau \in A_{i}} R\left(\tau, e_{i}\right)\right)
$$

for $i \in\{1,2\}$, therefore $L\left(e_{1}\right)(x) \neq L\left(e_{2}\right)(x)$.

Once we can decide equivalence in quadratic space, we can produce a bisimulation proof of equivalence in the same amount of space. We first produce the deterministic automata $M_{e_{1}}$ and $M_{e_{2}}$ equivalent to $N_{e_{1}}$ and $N_{e_{2}}$. The states of $M_{e_{1}}$ and $M_{e_{2}}$ are represented by the powersets of $\operatorname{dom} e_{1}$ and $\operatorname{dom} e_{2}$, respectively. These sets are of exponential size, but they can be generated sequentially in linear space. The transition function is the action on subsets as defined in Sect. 15.3.4, and this can also be generated in linear space.

Now we attempt to construct the maximal bisimulation between the two deterministic automata. We iterate through all pairs of states, testing equivalence of each pair as described above. If the states are equivalent, we output the pair as bisimilar. The set of pairs that are ever output is the maximal bisimulation.

In case $e_{1}$ and $e_{2}$ are not equivalent, a witness for inequivalence can also be produced in PSPACE. A witness for inequivalence is a guarded string $x$ accepted by 
one automaton but not the other. The shortest such string can be exponentially long in the worst case, but can be produced in the same way that one would produce an exponential-length accepting computation of a nondeterministic linear-space Turing machine, by a straightforward modification of the proof of Savitch's theorem (Savitch 1970).

Acknowledgements Many thanks to Jiř́ Adámek, Marcello Bonsangue, Helle Hvid Hansen, Raul Leal, Jan Rutten, Mehrnoosh Sadrzadeh, Luigi Santocanale, Alexandra Silva, Yde Venema, and James Worthington. An earlier version of this work (Kozen 2008) was presented at CMCS 2008 and was supported by NSF Grant CCF-0635028. The preparation of this revised version was supported by NSF grants CCF-1535952 and CCF-1637532 and by the National Security Agency.

\section{References}

Bonsangue, M. M., Rutten, J. J. M. M., \& Silva, A. M. (2007). Regular expressions for polynomial coalgebras. Technical Report SEN-E0703, Centrum voor Wiskunde en Informatica, Amsterdam.

Bonsangue, M. M., Rutten, J. J. M. M., \& Silva, A. M. (2009). A Kleene theorem for polynomial coalgebras. In L. de Alfaro (Ed.), Proceedings of the 12th international conference foundations of software science and computation structures (FoSSaCS 2009) (Vol. 5504, pp. 122-136)., of lecture notes in computer science New York: Springer.

Brzozowski, J. A. (1964). Derivatives of regular expressions. Journal of the Association for Computing Machinery, 11, 481-494.

Chen, H., \& Pucella, R. (2003). A coalgebraic approach to Kleene algebra with tests. Electronic Notes in Theoretical Computer Science, 82(1),

Cohen, E., Kozen, D., \& Smith, F. (1996). The complexity of Kleene algebra with tests. Technical Report TR96-1598, Computer Science Department, Cornell University.

Conway, J. H. (1971). Regular algebra and finite machines. London: Chapman and Hall.

Kaplan, D. M. (1969). Regular expressions and the equivalence of programs. Journal of Computer and System Sciences, 3, 361-386.

Kleene, S. C. (1956). Representation of events in nerve nets and finite automata. In C. E. Shannon \& J. McCarthy (Eds.), Automata studies (pp. 3-41). Princeton, NJ: Princeton University Press.

Kozen, D. (1994). A completeness theorem for Kleene algebras and the algebra of regular events. Computing and Information, 110(2), 366-390.

Kozen, D. (1997). Kleene algebra with tests. Transactions on Programming Languages and Systems, 19(3), 427-443.

Kozen, D. (2000). On Hoare logic and Kleene algebra with tests. Transactions on Computational Logic, 1(1), 60-76.

Kozen, D. (2003). Automata on guarded strings and applications. Matématica Contemporânea, 24, $117-139$.

Kozen, D. (2008). On the coalgebraic theory of Kleene algebra with tests. Technical Report.? http:// hdl.handle.net/1813/10173, Computing and Information Science, Cornell University.

Kozen, D., \& Smith, F. (1996). Kleene algebra with tests: Completeness and decidability. In D. van Dalen \& M. Bezem (Eds.), Proceedings of the 10th international workshop computer science logic (CSL'96) (Vol. 1258, pp. 244-259)., of lecture notes in computer science Utrecht: Springer.

Rutten, J. J. M. M. (1998). Automata and coinduction (an exercise in coalgebra). Proceedings of CONCUR'98 (Vol. 1466, pp. 193-217)., lecture notes in computer science Berlin: Springer.

Savitch, W. (1970). Relationship between nondeterministic and deterministic tape complexities. Journal of Computer and System Sciences, 4(2), 177-192.

Worthington, J. (2008). Automatic proof generation in Kleene algebra. In R. Berghammer, B. Möller, \& G. Struth (Eds.), 10th International conference relational methods in computer science (RelMiCS10) and 5th international conference applications of Kleene algebra (AKA5) (Vol. 4988, pp. 382-396)., of lecturer notes in computer science Berlin: Springer. 